\title{
PROCESS ORIENTED PRESENTATION OF MODELLING METHODOLOGIES
}

\author{
K. Kosanke \\ CIMOSA Association (e.V.) \\ Stockholmer Str. 7, D-71034 Böblingen, Germany, \\ Tel (49) 7031277665 Fax (49) 7031276698 e-mail ko@ipa.fhg.de
}

\begin{abstract}
Enterprise integration and enterprise modelling are gaining more and more importance in manufacturing engineering. However to guide the user through the rather complex tasks of enterprise integration and modelling both require sufficient methodologies. Methodologies in this area are very much process oriented following the life cycle concept which is very commonly used in systems engineering and product development. However, different methodologies place emphasis on different life cycle phases without identifying this emphasis explicitly. Process representation of enterprise integration and modelling methodologies enables identification of their compatibility and differences. Using CIMOSA as the modelling method process models have been created for several enterprise modelling methodologies.

The paper intends to demonstrate the benefits of a common process oriented representation of modelling methodologies. It does not claim completeness and full correctness of the process models presented. Further work will be needed to capture all the details of the textual descriptions available today.
\end{abstract}

\section{Keywords}

Modelling Methodologies, Enterprise Integration, Enterprise Modelling, Process Modelling, CIMOSA.

\section{INTRODUCTION}

Methodology is the system of methods and principles used in a particular discipline and method is a way of proceeding or doing something; the technique or arrangement of work for a particular field (Collins, 1987). This definitions implies the process nature of methods and therefore methodologies as well. Process representations, especially graphical ones, are much more easily understood and comparable with each other. In addition, most Methodologies are based on the system life cycle concept which allows a comparison of the different methods in terms of the coverage of different process steps in the life cycle. For a comparison of different methodologies see also Williams, et al (1994) and which reports on the results of the IFAC/IFIP Task Force on Architectures for Integrated Manufacturing Activities and Enterprises. The Task Force compared three different methodologies CIMOSA ${ }^{1}$, GRAI $^{2}$ and PERA ${ }^{3}$.

$1 \quad$ Open System Architecture for CIM

2 Graphs with Results and Activities Interrelated (Graphe à Résultant et Activités Interreliés) 
This paper presents a process representation of several enterprise modelling methodologies, using a graphical representation of the process models of different methodologies which is based on CIMOSA (ESPRIT Consortium AMICE, 1993) an ESPRIT supported development.

The different methodologies represented and compared are CIMOSA, IEM(IUM) ${ }^{4}$ and PERA (GRAI tbd). The work is based on material describing the different methodologies available to the author. It represents the authors view of the methodologies and may be modified in the course of further discussions with the developers and owners of the methodologies themselves. Due the limitations of a paper the graphical representation of the different methodologies is limited to examples of those processes rather than providing the complete process description. In addition, the paper analyses the modelling language (modelling constructs) used in the different methodologies using the CIMOSA language as a reference. Details of the information used and produced in the course of the modelling process has also been done but is not presented in the paper. The identification of information used and produces in the different activities allows to identify and provide/eliminate missing or redundant information and no value information, respectively.

In the following the modelling methodologies are described in terms of their information exchange with the environment (the latter is describes as non-CIMOSA Domains) in the methodology overviews and their internal process structure (identified as the CIMOSA Domain) in the additional Figures provided for each methodology. The terms CIMOSA Domain and noneCIMOSA Domain mean the internals of the domain are either further detailed into a process model according to CIMOSA or are left without considering the internal structure of that domain. The different processes (DP $=$ Domain Process) identified for the methodologies (CIMOSA Domain) correspond to the different phases of the system life cycle. These processes are further detailed into either sub-processes $(\mathrm{BP}=$ Business Process) or activities $(\mathrm{EA}=$ Enterprise Activity). Behavioural Rules define the process flow (control flow) identifying the conditions for continuation after ending an activity. Due to the time independence of the domain processes the process flow between those processes themselves is defined by events rather than behavioural rules.

A comparison of the different methodologies is presented at the end of the paper in Table 1. For more information on CIMOSA representation see references ESPRIT Consortium AMICE (1993) and (1994).

\section{PERA (PURDUE ENTERPRISE REFERENCE ARCHITECTURE)}

The PERA modelling methodology is described in numerous publication (Williams, 1994) and $\mathrm{Li}$, H. and Williams, T. J. (1994). PERA is intended to support and guide the development of the master plan for an enterprise business entity. The methodology covers the complete system life cycle starting with the definition of the business entity to be modelled, identifying its mission, vision, management philosophy, mandates, etc. and ends with obsolescence of the plant at the end of the operational phase. Its life cycle phases are described for both personnel and information and product and operational requirements leading to an information architecture, an human and organisational architecture and a manufacturing equipment architecture (see Figure 1).

\subsection{PERA Modelling Constructs}

The PERA modelling constructs are represented in Figure 1. PERA like CIMOSA provides for identification of the entity to be modelled and of the activities to be carried out in the course of the modelling process. CIMOSA on the other hand provides explicit recognition of the environment of modelling itself and a representation of the process dynamics (events and behavioural rules), distinguishing between process and activity. Both methodologies provide for different views on the model as shown in the lower part of Figure 1. PERA identifies three different architectures and CIMOSA allows for four different views on the model of the particular enterprise.

4 Integrated Enterprise Modelling (Integrierte Unternehmens-Modellierung) 


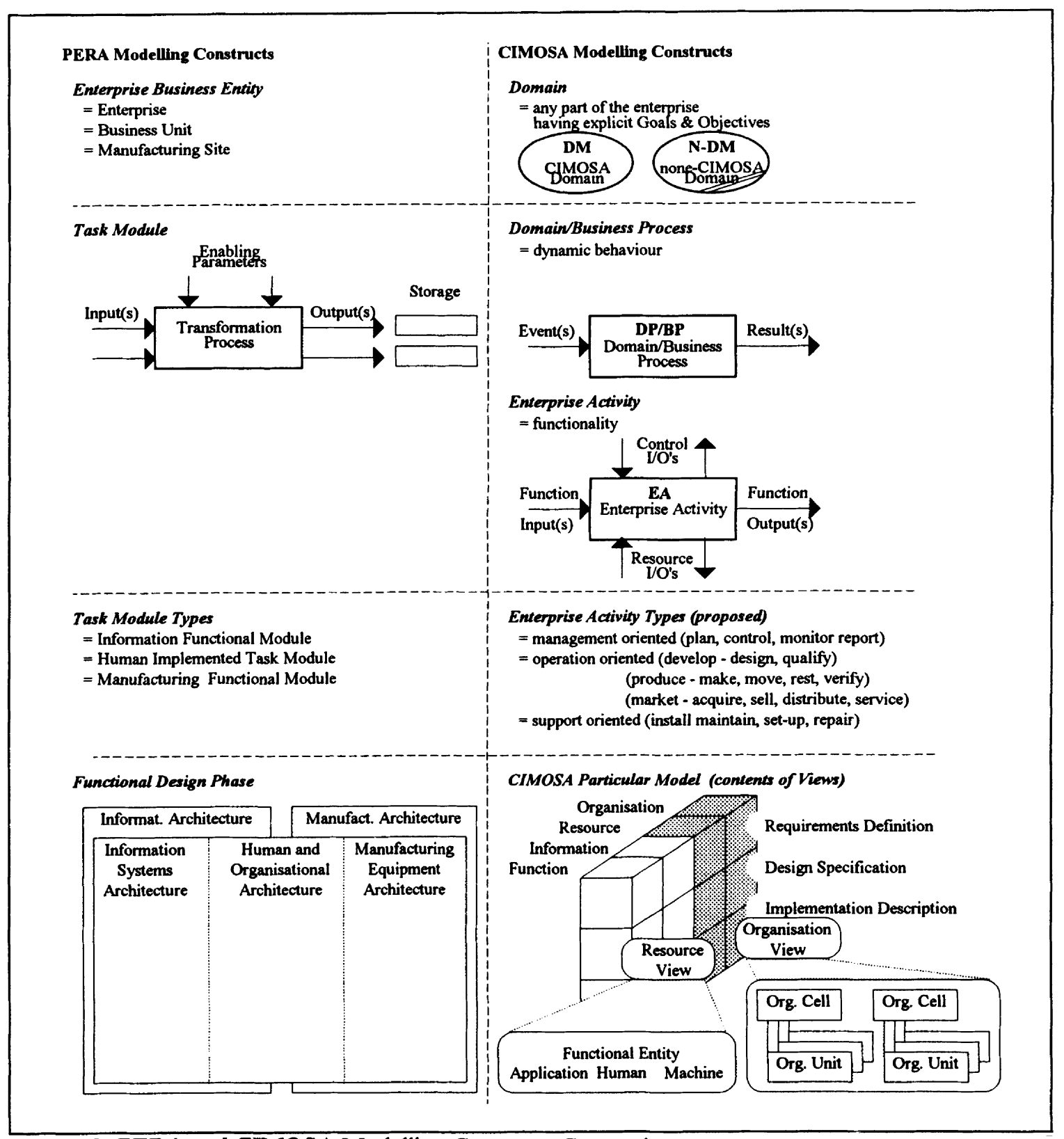

Figure 1: PERA and CIMOSA Modelling Construct Comparison

\subsection{PERA Modelling Methodology - Process Overview}

The following is an attempt to establish a process model of the Purdue Enterprise Reference Architecture methodology using the CIMOSA modelling language (CIMOSA modelling constructs). A draft of the process model is provided which has been developed in co-operation with T. Williams and co-workers. The modelling environment overview (Figure 2) provides the relation between the further detailed CIMOSA Domain 'Enterprise Business Entity Master Plan Development' and three none-CIMOSA Domains. Information exchange is identified on a rather high level indicating global object views and events exchanged between the different domains. However no further details of the information exchange are currently identified in this modelling approach of PERA 


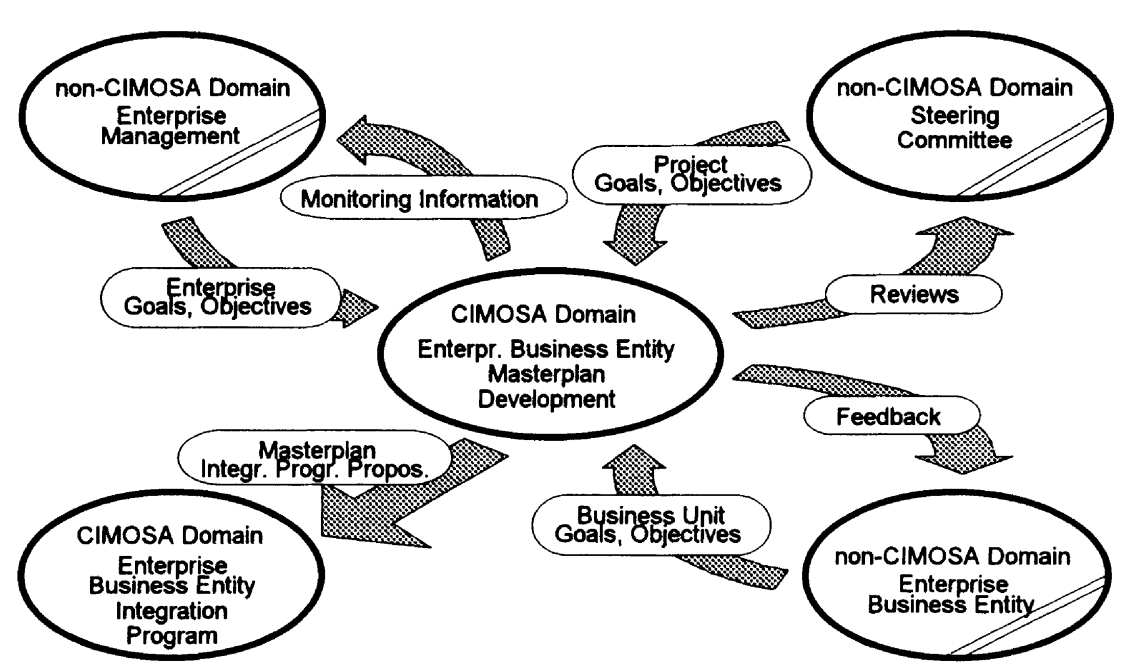

Figure 2: PERA Methodology Overview - Enterprise Business Entity Masterplan Development Project and its Relation to other Domains

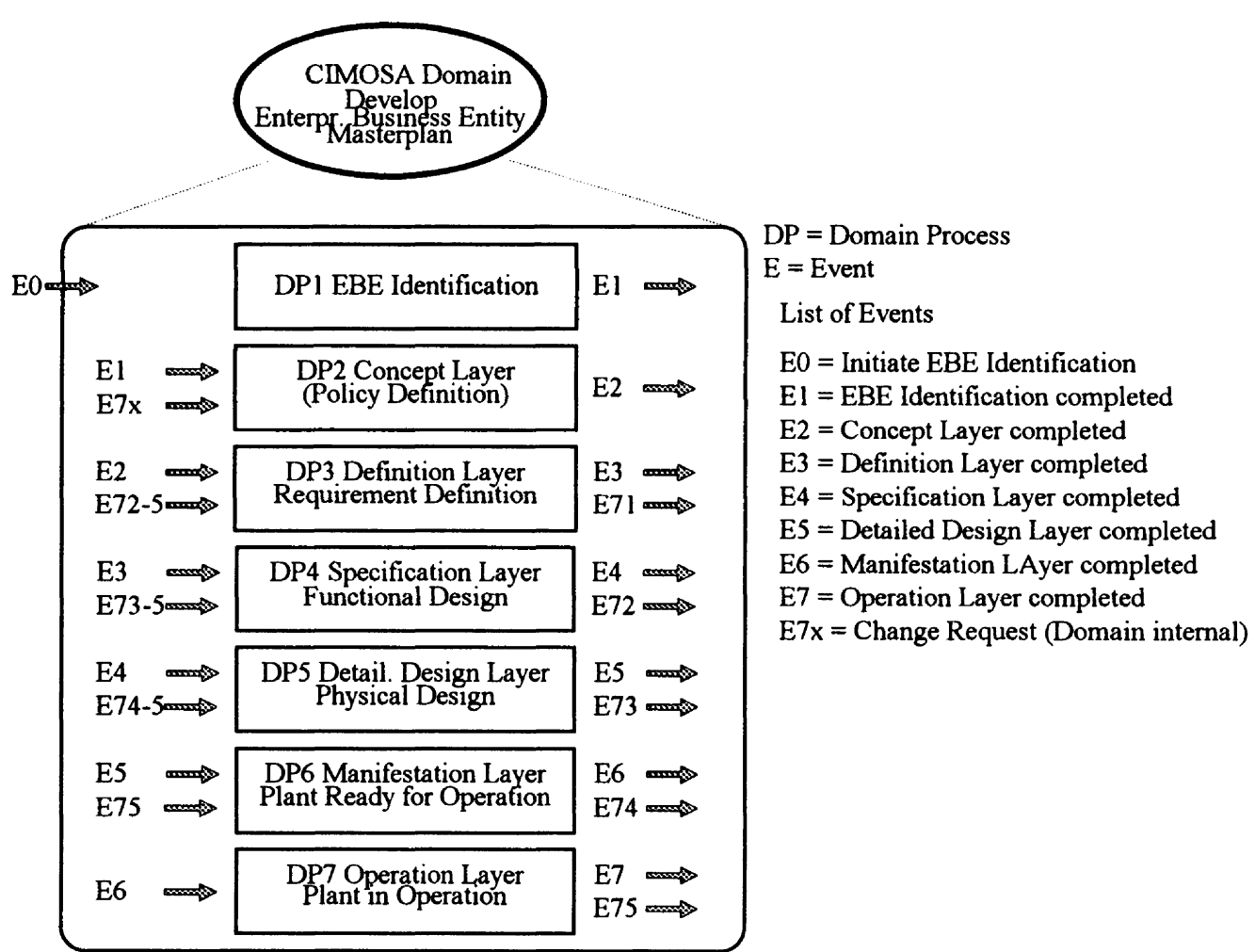

Figure 3: PERA Modelling Methodology - Domain Processes

The details of the PERA modelling methodology (CIMOSA Domain) are shown in Figure 3. Seven domain processes have been defined covering each one of the different phases of the system life cycle identified in the layering diagram of the PERA methodology. Enterprise events have been defined which enable the co-operation of the different domain processes indicating completion of 
processes or needs for changes of results of previous ones. Figure 4 provides an example of the details of the different domain processes represented on business processes and enterprise activity level. The example shows the parallel efforts for the three architectures of PERA for information, human and organisation and manufacturing equipment. Behavioural rules are only indicated but are not further defined.

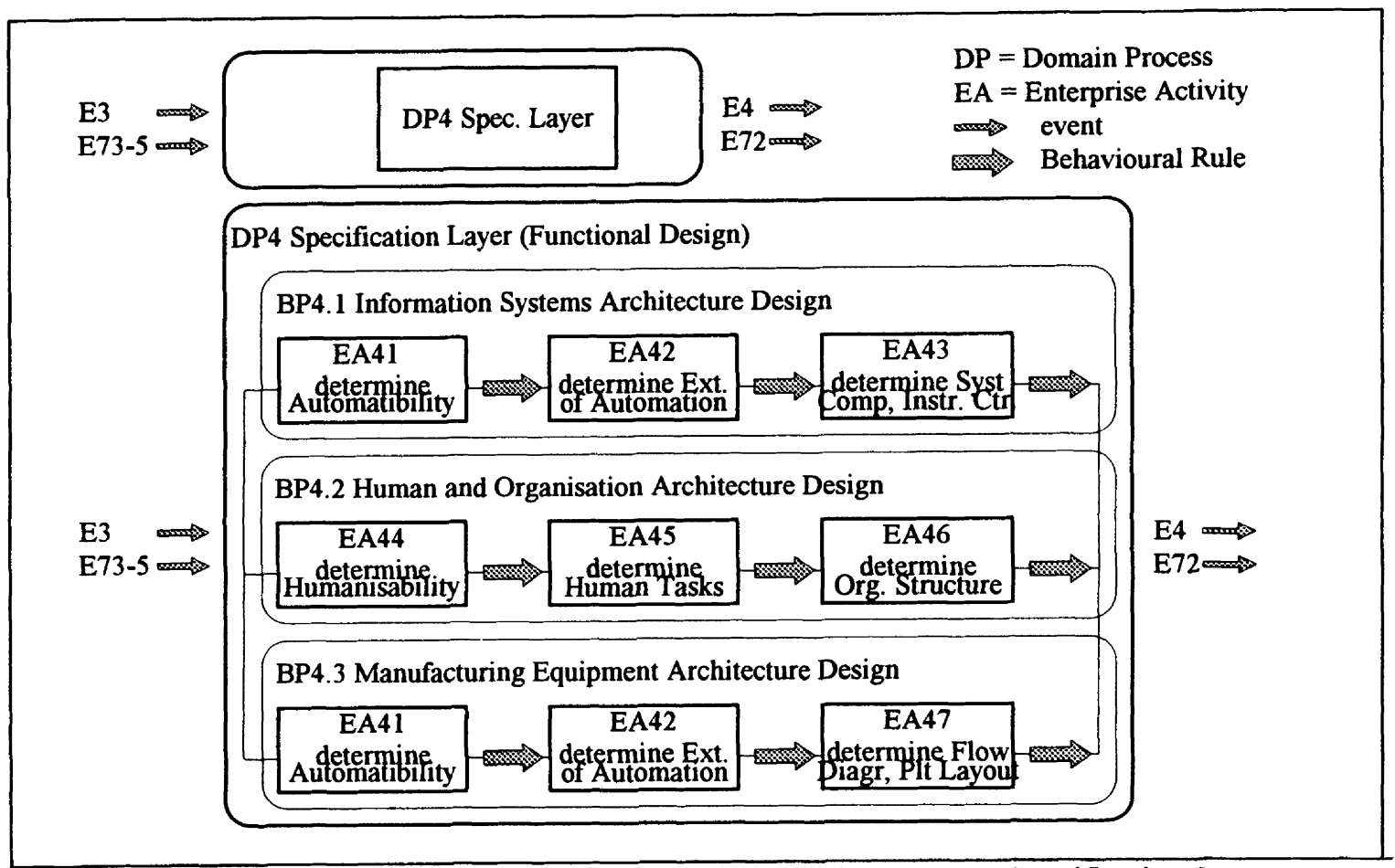

Figure 4: PERA Modelling Methodology - Details of Domain Process - Specification Layer

\section{CIMOSA (CIM OPEN SYSTEMS ARCHITECTURE)}

CIMOSA models are intended to be used for operational support rather than as project guides in developing or re-engineering business entities only. Operational use is seen as decision support for evaluating operational alternatives as well as model driven operation control and monitoring. CIMOSA supports the engineering of enterprise models from requirements definition to implementation description, their operational use and maintenance employing the system life cycle concept as well. The life cycle coverage is less than of PERA leaving out the definition of the business entity and the operational phase. CIMOSA assumes business objectives and constraints to be provided by management and operation itself not to be part of the modelling process. It also identifies model maintenance as a specific phase of the life cycle rather than being part of operation as in PERA.

To support the business user in his two roles of engineering and maintaining the model and using the model in support of his operational task CIMOSA provides four views of the actual enterprise model. This enables the user to focus on his particular interest rather than being confused by the complexity of the complete model. The modelling methodology of CIMOSA is described in a recent publication (Zelm, et al, 1995).

\subsection{CIMOSA Modelling Constructs}

Figure 5 shows the set of business modelling constructs of CIMOSA which is used in the comparison. The CIMOSA meta model defines the structure of modelling constructs and identifies object classes and elements of the modelling languages. The classification provides for the selection of different views on the model with Function View for representation of process dynamics 
(processes, events) and functionality (domain, enterprise activity), Information View for information management (enterprise object), Resource View for resource management (capability set, resource) and Organisation View for organisation representation (organisation cell and unit) and identification of responsibilities and authorisation on the different objects in the enterprise (processes, information, resources and organisation itself).

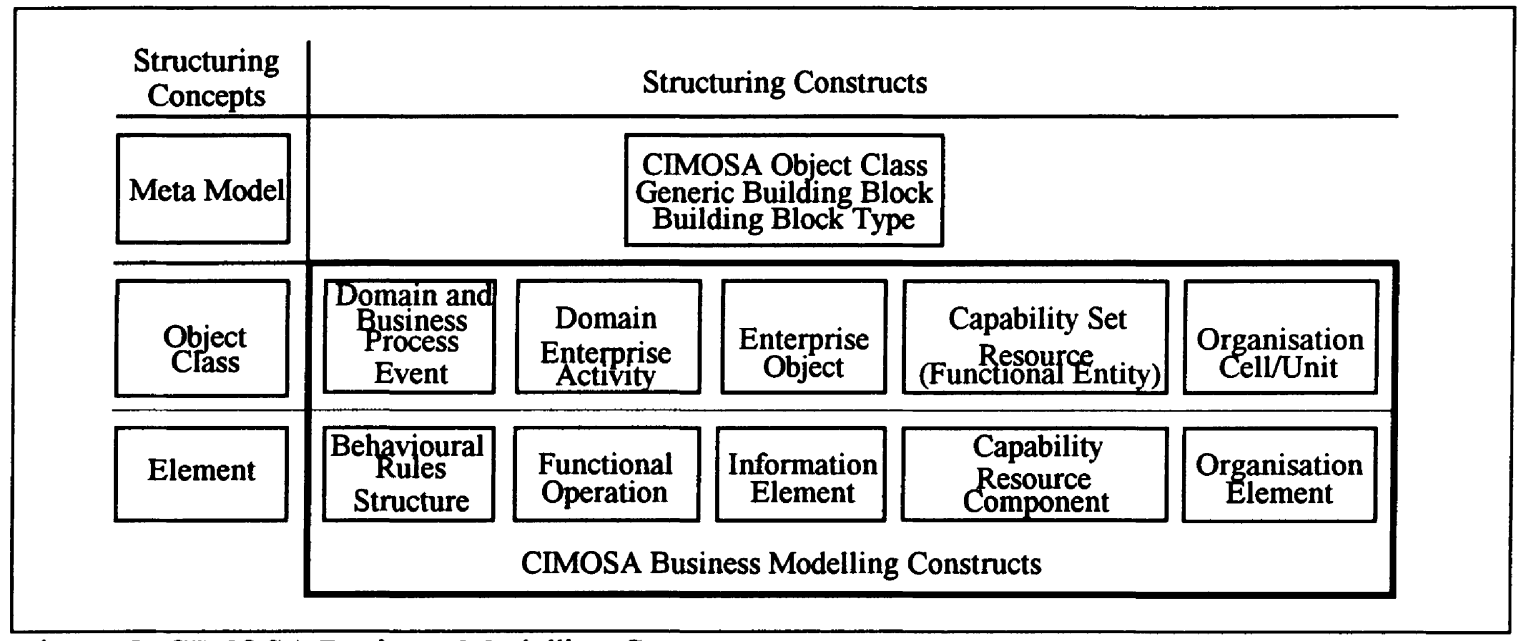

Figure 5: CIMOSA Business Modelling Constructs

\subsection{CIMOSA Modelling Methodology - Process Overview}

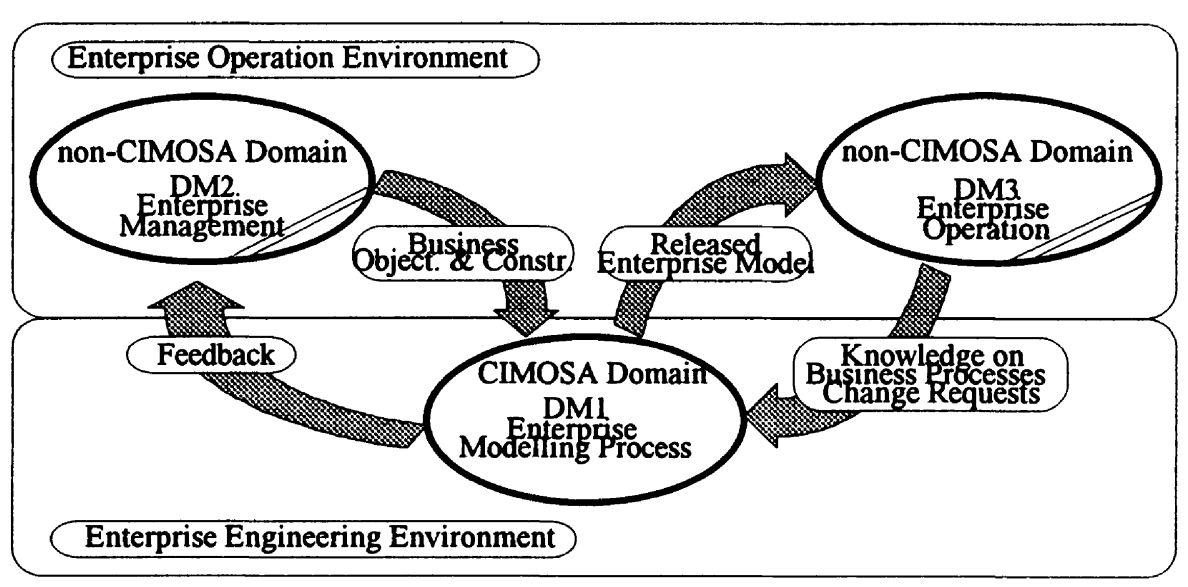

Figure 6: CIMOSA Methodology Overview - CIMOSA Business Modelling Process

An overview of the CIMOSA modelling process environment is shown in Figure 6. Due to the different goals of CIMOSA and PERA modelling, the relations to the environment are less complex for CIMOSA modelling methodology involving only enterprise management and the relevant part of the enterprise operation. The relation between the further detailed CIMOSA Domain 'Enterprise Modelling Process' and two none-CIMOSA Domains are described as information exchange on a rather high level indicating global object views and events exchanged between the different domains.

The details of the CIMOSA modelling process are shown in Figure 7. Four domain processes have been defined covering the different phases of the system life cycle identified in the CIMOSA methodology. Enterprise events have been defined which enable the co-operation of the different 
domain processes indicating completion of processes or needs for changes of results of previous ones.

Figure 8 provides an example of the details of the different domain processes represented on business process level only. The example shows the efforts relating to the different model views of CIMOSA. The first part is concerned with the function view whereas the three parallel processes use the information, resource and organisation view capabilities, respectively.

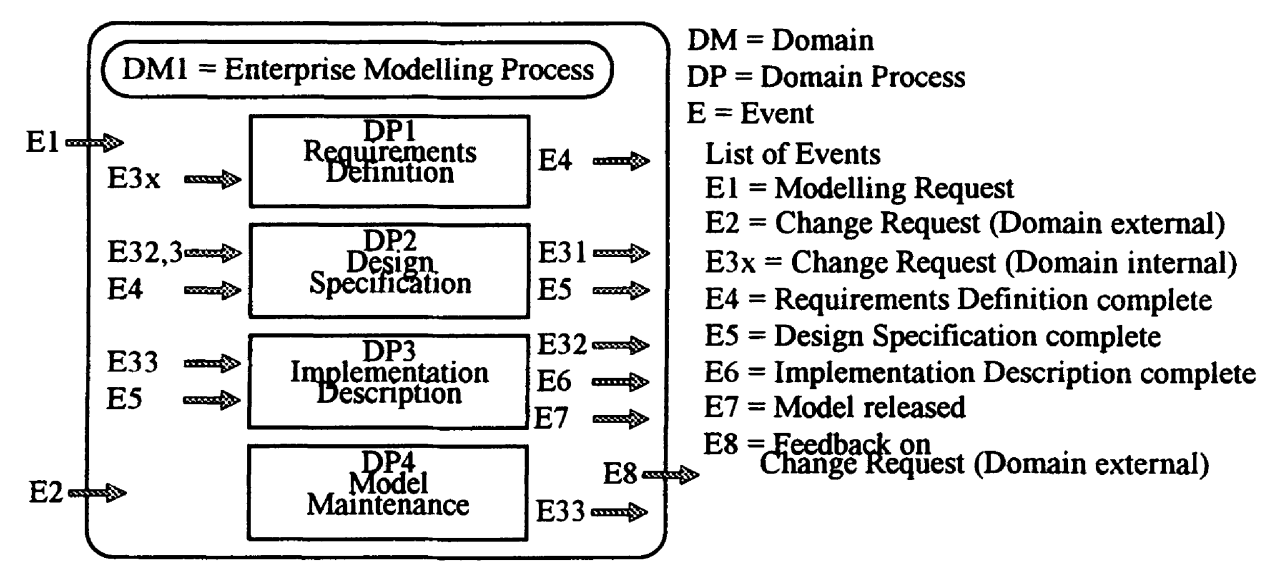

Figure 7: CIMOSA Business Modelling Process - Domain Processes

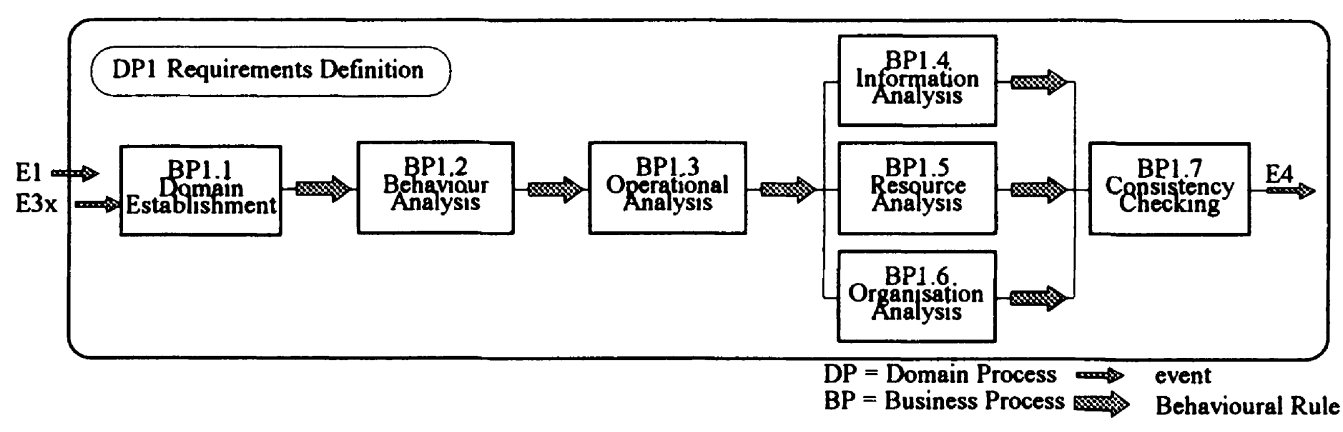

Figure 8: CIMOSA Business Modelling Process - Details of Domain Process - Requirements Definition

\section{IEM (INTEGRATED ENTERPRISE MODELLING)}

The IEM modelling methodology supports creation of enterprise models for business reengineering and therefore allows also to model process dynamics for evaluation of operational alternatives. System life cycle support is rather limited at its later phases. Focus is on requirements modelling with some efforts on system design and implementation description. The definition of business goals and objectives is also not covered by the IEM methodology.

\subsection{IEM Modelling Constructs}

The modelling constructs of IEM are shown in Figure 9. IEM defines its constructs for the descriptions of functionality in three different stages of completeness. It identifies three inputs and one output on its activity construct compared with CIMOSA which provides additional outputs for 
control and resource status information. Similarly IEM provides two different views on its processes using two of the three different representations of functionality. This allows to show control and resources directly in the process representation providing a combined view of product flow (process flow), its control through orders and the use of the resources needed in the production process. The two sets of modelling constructs for describing the process dynamics only differ in their terminology.

A very extensive comparison between IEM and CIMOSA modelling constructs has been made jointly by the two originating teams from IPK, Germany and CIMOSA Association trying to converge to a common modelling language. This comparison is described in a joined paper submitted to the European standardisation (CEN TC 310/WG1 1995).

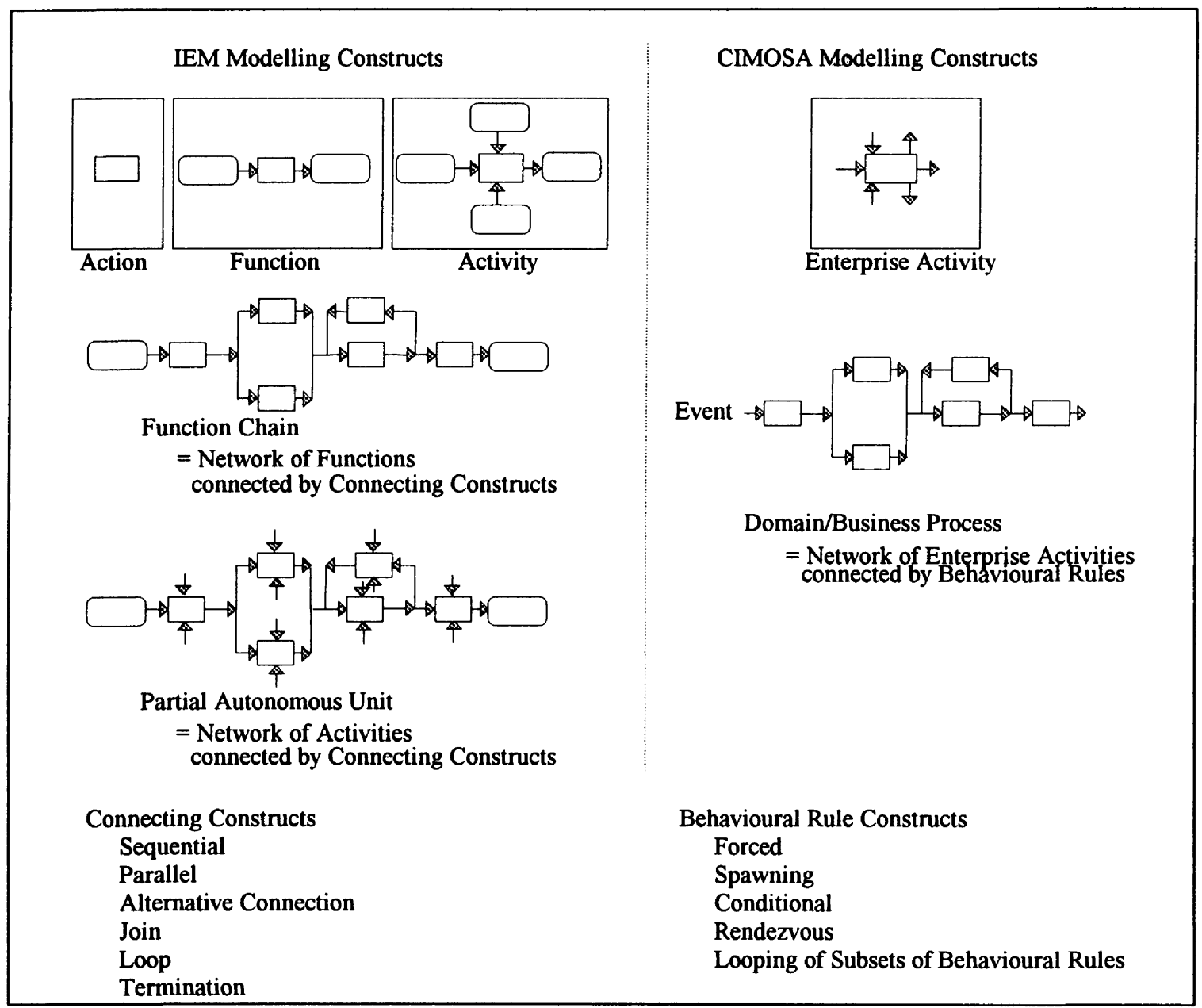

Figure 9: IEM and CIMOSA Modelling Construct Comparison

\subsection{IEM Modelling Methodology - Process Overview}

Only the overview of the IEM modelling methodology is shown in Figure 10. Again four phases of the system life cycle are covered by the IEM methodology, however, with much limited details at its later phases.

An example of the details of the different domain processes represented on only business processes level is shown in Figure 11. At this level of requirements definition IEM identifies more tasks than any of the other methodologies considered. Emphasis is placed on a much more specific description of the requirements in order to enable model execution for simulation. CIMOSA 
foresees this kind of model use only at the design specification modelling level. The example shows the modelling tasks relating to the different types of objects identified for distinguishing product, order and resource information to be used in the IEM process representation (Partial Autonomous Unit).

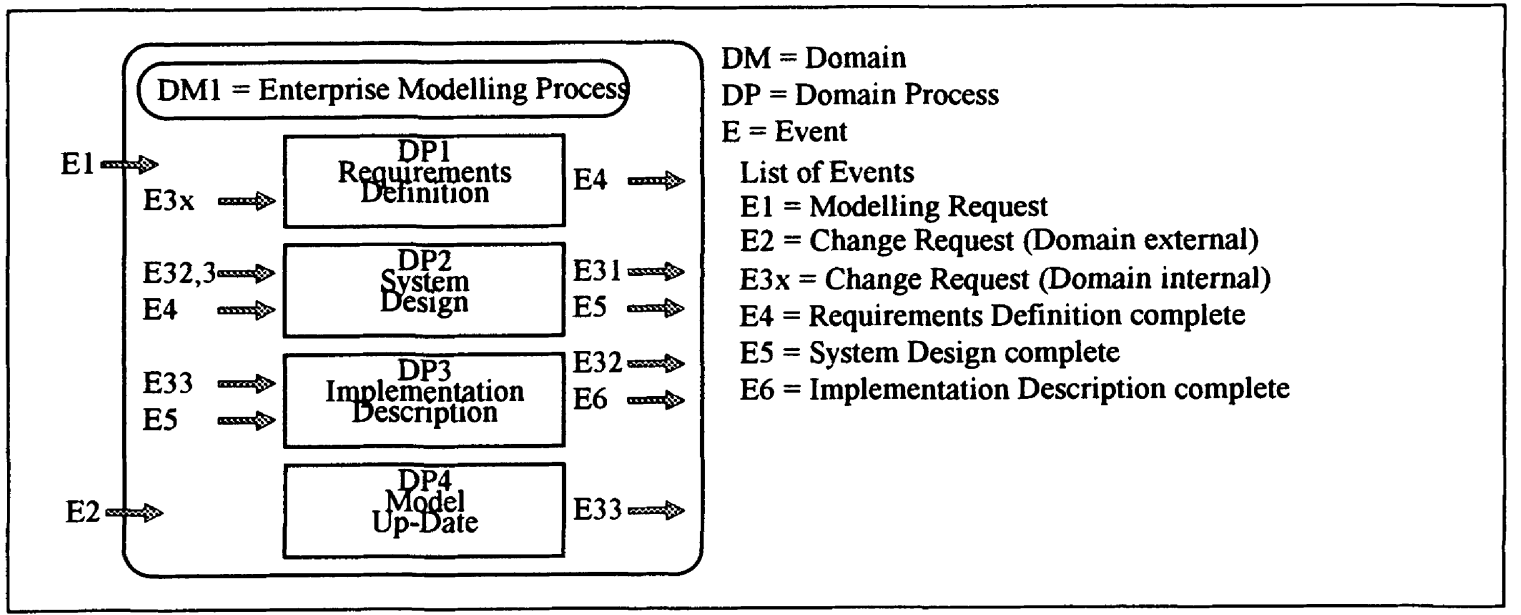

Figure 10: IEM Modelling Methodology - Domain Processes

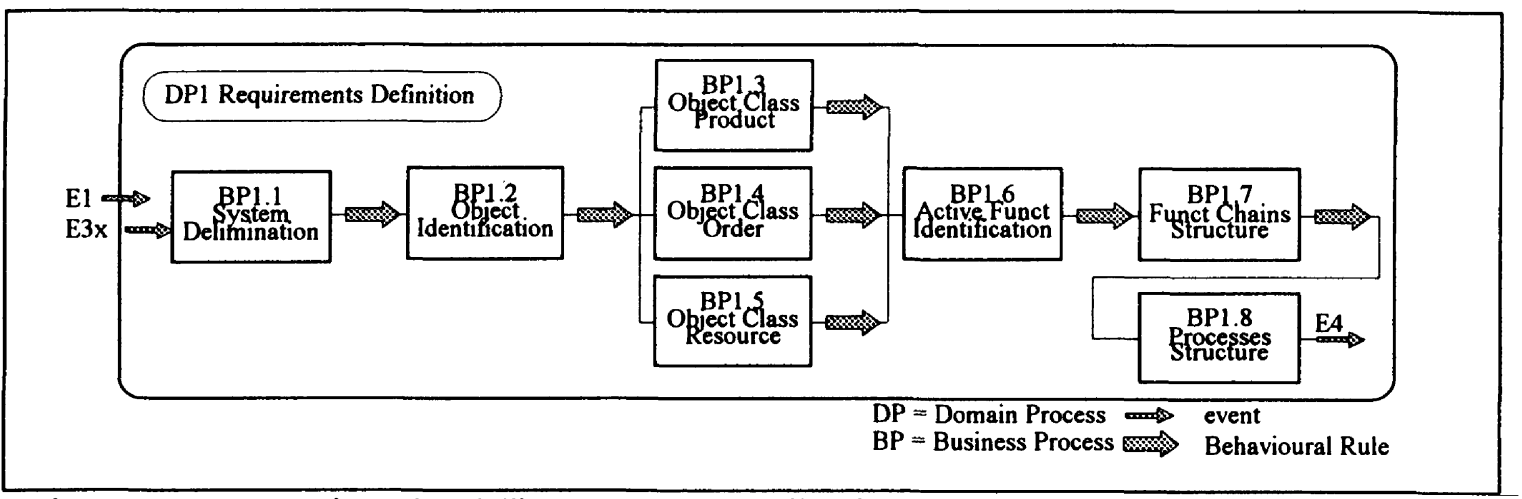

Figure 11: IEM Business Modelling Process - Details of Domain Process - Requirements Definition

\section{METHODOLOGY COMPARISON PERA - CIMOSA - IEM}

Table 1 shows the process models of the three methodologies. The representation follows the system life cycle concept identified for the PERA methodology adding the maintenance phase of CIMOSA.

Using the domain process definitions given for the different methodologies as a representation of the life cycle phases their complete structures in terms of business processes and identification of lower level enterprise activities are presented in relations to each other. The lower level is not yet available for the IEM modelling methodology. The number of enterprise activities identified for PERA and CIMOSA are 48 and 77 respectively. 


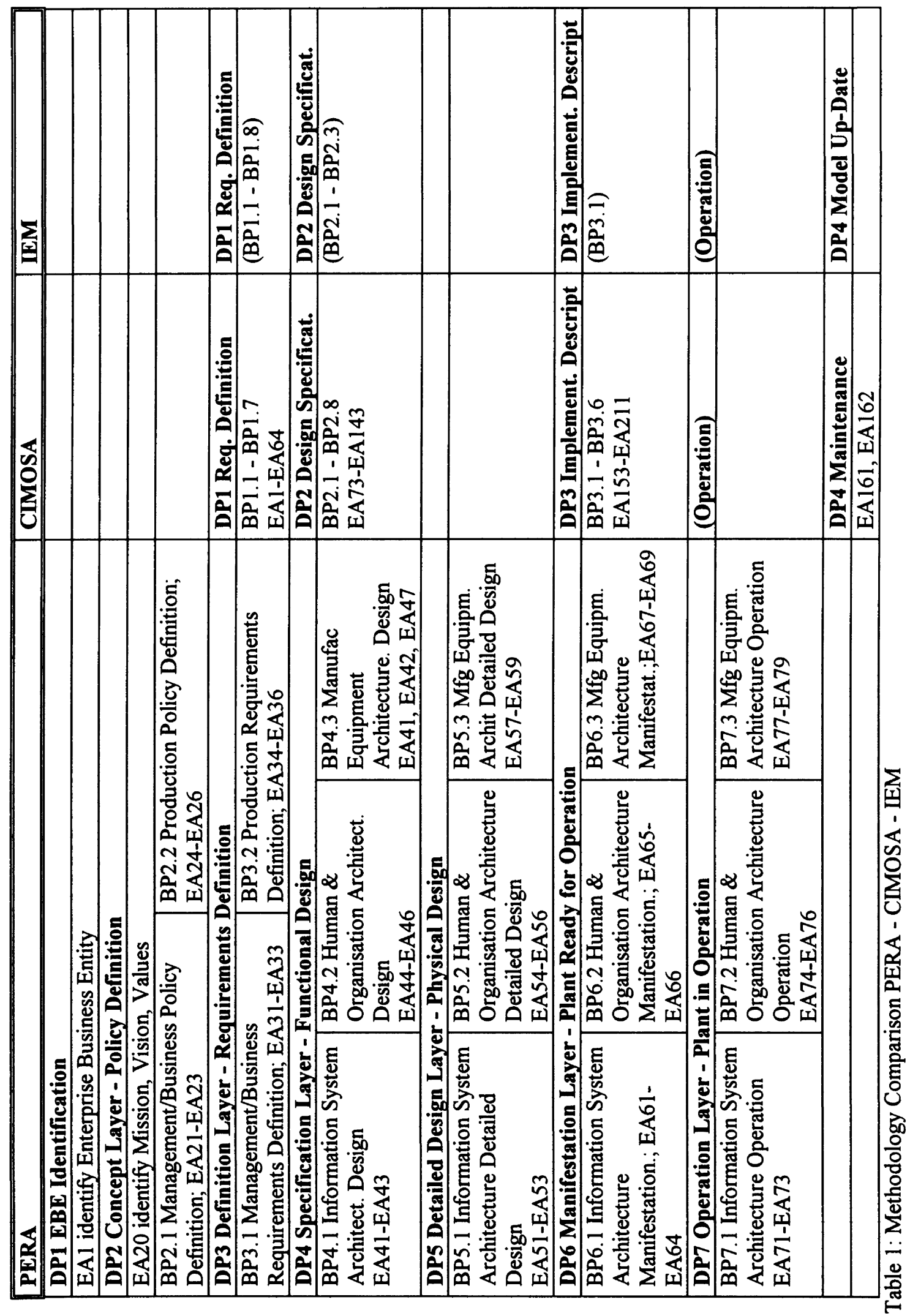


The PERA methodology shows the highest coverage of the life cycle phases, starting with identification of the business unit itself and its strategic role and objectives and ending with enterprise operation. The other two methodologies assume the enterprise strategy to exist and only identify operational requirements derived from given objectives and constraints. However both IEM and CIMOSA place much more emphasis on the requirements phase with 8 and 7 business processes, respectively, identified for this phase compared with 2 for the PERA methodology. IEM has its focus on the requirements phase where it identifies the highest number of tasks on the business process level.

PERA places more emphasis on the design of the operational system distinguishing explicitly between functional and physical design. Its including of the operational phase indicates the emphasis on enterprise integration rather than on enterprise modelling. CIMOSA on the other hand places its emphasis on enterprise modelling explicitly defining the tasks of model maintenance as part of its modelling methodology. Model maintenance is seen as part of the operational phase in PERA. Model up-date is mentioned in IEM but is not identified as a modelling task (yet).

\section{CONCLUSIONS}

This comparison demonstrates the advantage of the process oriented presentation of the modelling methodologies for comparing different methodologies. The structuring of the methodologies into process tasks provides visibility on the emphasis placed on the different parts of the life cycle. It will also enable users to select or even combine methodologies according to their needs and may get developers to provide additional tasks in their own methodology.

The work indicates the wide overlap between enterprise integration and enterprise modelling methodologies. Enterprise modelling is a major part of enterprise integration. It really becomes the enabling technology for enterprise integration by providing visibility on the enterprise operation. Enterprise models established according to a user oriented, consistent and accepted methodology can be used in decision support for the evaluation of operational alternatives or even for model driven operation control and monitoring. However, to really become an efficient and effective decision and operational support tool, enterprise modelling methodologies have really to be implemented in computerised tools. Tools which provide a sufficient user interface to enable the operational staff to use and maintain enterprise models in their day-to-day tasks in the enterprise.

More work is still required on the contents of the different methodologies to make them consistent in themselves. Also identification of the information used and produced in the course of model creation is still not complete. Additional benefits will be obtained by taking advantage of the common representation and to converge terminology and task definition.

\section{REFERENCES}

Collins (1987) Dictionary and Thesaurus. Collins, London \& Glasgow.

Williams, T. J. et al (1994) Architectures for integrating manufacturing activities and enterprises. Computers in Industry. 24/2-3, 111.

$\mathrm{Li}, \mathrm{H}$. and Williams, T. J. (1994) A Formalization and Extension of the Purdue Enterprise Reference Architecture and the Purdue Methodology. Report Number 158 Purdue Laboratory for Applied Industrial Control

ESPRIT Consortium AMICE (1993) CIMOSA - Open System Architecture for CIM. SpringerVerlag, (ISBN 3-540-56256-7), (ISBN 0-387-56256-7).

CIMOSA Association (1994) CIMOSA - Open System Architecture for CIM; Technical Baseline", Version 3.0 October, private publication.

Williams, T. J. (1994) The Purdue Enterprise Reference Architecture. Computers in Industry, 24/2-3, 141 .

Zelm, M. et al. (1995) The CIMOSA Modelling Process. Computers in Industry, accepted for publishing

CEN TC 310/WG1 (1995) Document N 41.3: QCIM/WG QEM - ESPRIT CIMOSA, Comparison CIMOSA - IEM Modelling Constructs and Methodology. 